\title{
DESDE CUMANÁ HASTA SANTA ANA. LA RELACIÓN DE AIMÉ BONPLAND CON LAS AMÉRICAS
}

\author{
From Cumaná to Santa Ana. Aimé Bonpland's relashionship with Americas
}

\author{
CÉDRIC CERRUTI'P
}

\begin{abstract}
Resumen: La relación de Aimé Bonpland con las Américas invita a reflexionar sobre las condiciones necesarias para la transferencia de una ciencia y sobre la ideología que la sustenta. Partidario de la independencia del Río de la Plata, inmerso en una cultura política que pone en primer plano la necesidad de un Estado fuerte que permita la estabilidad y la influencia de una nación, Bonpland se enfrenta a la inestabilidad que caracteriza la construcción política de esta región. Al seguir su camino, asistimos a la reunión entre un naturalista que lleva a cabo un proyecto basado en el modelo universalista de la ciencia europea y las élites rioplatenses que desean implementar una política específica basada en el uso de los recursos naturales con el propósito de desarrollo interno. También estamos presenciando una transformación de un viajero en un migrante.
\end{abstract}

Palabras clave: Americanismo, historia natural, movimientos independentistas.

Summary: Bonpland's relashionship with Americas leads us to reflect about the necessary conditions for the transfer of a science and the ideology it bears. In favour of the independence of the Rio de la Plata, imbued with a political culture that puts forward the necessity of a strong state thus enabling a nation to be stable and to shine forth, Bonpland faces the instability that characterizes the political construction of this region. By following his path, we witness the union of a naturalist leading a project based on the universalist pattern of the European science with the Rio de la Plata elites who want to implement a specific policy based on the use of natural resources for internal development. We are also witnessing a transformation of a traveler into a migrant.

Key words: Americanism, independence movements, natural history.

\section{Introducción}

Desde el puerto de Cumaná donde desembarcó por primera vez en América con Alejandro von Humboldt el 16 de julio de 1799, hasta la estancia de Santa Ana (actual localidad de Bonpland, Paso de los Libres, Corrientes) donde falleció cerca del río Uruguay el 10 de mayo de 1858, Aimé Bonpland desarrolló una relación muy estrecha con las Américas.
Médico, botánico, explorador, naturalista, terrateniente, agricultor, el científico multiplicó las actividades y se enraizó poco a poco en las tierras americanas.

En un primer tiempo hace falta recordar el "Grand Tour" americano llevado a cabo con Alexander von Humboldt entre 1799 y 1804 como poner de relieve los aportes de Bonpland durante la expedición común, para después analizar el contexto científico y

\footnotetext{
${ }^{1}$ Investigador asociado del CRHIA, Universidad de La Rochelle, 23 Avenue Albert Einstein, 17000 La Rochelle, France. E-mail: cedriccerruti@yahoo.es
} 
político dentro de lo cual Bonpland emprendió otro viaje a América. En segundo lugar, examinaré su trayecto desde su partida de Francia en 1816 hasta 1858 para destacar la relación que Bonpland desarrolló con el Sur de América a través de las interacciones políticas o científicas, como las dificultades que existieron para establecer vínculos científicos trasatlánticos en esa época.

\section{Felices coincidencias (1798-1816)}

La expedición americana de Humboldt y Bonpland fue una "feliz coincidencia" porque el destino previo no era América sino un viaje alrededor del mundo. Fueron los eventos políticos quienes decidieron a Humboldt y Bonpland a dar rumbo hacia España. Cuando llegaron a Madrid en febrero de 1799, se encontraron con un contexto ilustrado y científico favorable pues la monarquía española había dedicado durante la segunda mitad del siglo XVIII un presupuesto incomparable al desarrollo científico.

En efecto, Carlos III (1759-1788) fue, a semejanza de su primo francés Luis XVI, un gran defensor de las ciencias. Carlos IV (17881808) no fue tan amante de las ciencias como su padre, ni tan reformista. En el contexto de la separación de las colonias inglesas de América, y sobre todo quebrantado por la revolución francesa, el reinado de Carlos IV se caracterizó por un clima de inestabilidad y de enfrentamientos entre los diversos partidos de la Corte madrileña.

Sin embargo, ese contexto favoreció a Humboldt y Bonpland. En efecto, Humboldt supo poner de relieve los beneficios que podía obtener la Corona de tal viaje. En efecto, Humboldt era especialista en minería, lo que era primordial para España. En cuanto a Bonpland, su papel como botánico era muy apreciable pues permitía no solamente seguir con la gran tradición hispánica de clasificación del reino vegetal, sino también añadir nuevas fuentes de riquezas medicinales o agrícolas en esa esfera de las ciencias naturales.

De esa manera se esperaba también un segundo beneficio: aprovechar las investigaciones de Humboldt y Bonpland para combatir la "leyenda negra" apoyándose en métodos, instrumentos y conclusiones científicas, probando así el valor como la riqueza de América. Por fin, Humboldt propuso redactar un balance científico y económico de las colonias en un periodo de crisis económica en la metrópoli, asumiendo todos los gastos desde la investigación hasta la edición (Gerbi, 1955; Rebok, 2003).

Ese contexto como los manejos diplomáticos de Humboldt permitieron obtener un pasaporte que no establecía límite alguno a sus investigaciones; por primera vez, las colonias se abrían a unos extranjeros sin ningún control institucional. Era un cambio total en la política española. Hace falta recordar que mientras Humboldt y Bonpland embarcaban en La Coruña para dar rumbo a América, en junio de 1799 , otro científico se encontraba en la ciudad encarcelado. Alejandro Malaspina, científico italiano, había llevado a cabo otra expedición científica gigantesca en América, cruzando el Pacífico hasta las Filipinas y las costas de China. Pero sus ideas liberales y reformistas hicieron imposible la publicación de los resultados, pues Malaspina se acercaba a la idea de que las colonias debían formar parte activa del sistema político europeo, con personalidad propia y plena capacidad para gestionar sus recursos.

Humboldt y Bonpland investigaron durante cinco años a través de Venezuela, Cuba, Colombia, Ecuador, Perú y México. Humboldt, el teórico de la expedición, desarrolló entonces en el laboratorio americano una nueva ciencia, la geografía política, es decir una investigación holística, global, universal que llevó a cabo tanto durante el viaje, como en la etapa posterior de estudio y elaboración de los resultados. Su visión transdisciplinaria de la ciencia no solamente estaba basada en los experimentos empíricos, sino también en los estudios de los archivos, de la prensa y de los libros a los cuales Humboldt y Bonpland tuvieron acceso sin límites. Les permitió valorar en su justa medida los cronistas o los científicos anteriores; valorar sus obras catalogadas como exageradas en Europa en ese momento (Rebok, 2003).

Humboldt se situó como el continuador de esos cronistas y científicos. Así, durante sus exploraciones, Humboldt y Bonpland recolectaron las muestras de la magnitud 
científica e ilustrada hispánica, participando en la recuperación ideológica del Nuevo Mundo. Un Nuevo Mundo hasta aquel momento despreciado. El enfoque científico de Humboldt y Bonpland fue un enfoque más neutral, sin contradicción posible porque hicieron hablar las cifras y los hechos probados científicamente.

Lo hicieron cooperando con las autoridades científicas y políticas que encontraron, y más bien lo hicieron con todas las personas que les ayudaron en sus exploraciones. Esa cooperación europea-americana se concretó por ejemplo en Quito o Bogotá donde los dos viajeros intercambiaron informaciones e ideas, cuándo se enfrentaron con los hitos políticos internos. Fueron recibidos por Juan Pío Montúfar en cuya residencia se alojaron y la cual le sirvió, además, para reunirse con jóvenes que años después participarán en la gesta independentista encabezada por Montúfar (Guerrero, 2019).

Medir el impacto de esos intercambios en el pensamiento de los criollos como en el pensamiento de Humboldt y Bonpland parece bastante difícil. Pero lo cierto es que fueron otra fuente de conocimiento hacia las ideas liberales que circulaban en Europa. Por parte de unas élites americanas, se podría evocar la "humboldteanización" (Zeuske, 2003) de su pensamiento, es decir la influencia intelectual que pudo tener Humboldt sobre esas élites. Pudiera ser el caso de Carlos Montúfar quien los acompañó durante su expedición desde 1802 hasta llegar a Europa, donde primero se puso a favor del movimiento reformista, siguiendo las ideas de Alexander von Humboldt, pues de regreso a América se puso en una postura política independentista visto que su padre fue un actor fundamental del movimiento (Hampe Martínez, 2002).

Con la publicación de los resultados de la expedición, los americanos encontraron a unos socios de sus reclamos. De la misma manera que el pintor Eduard Ender puso en escena la mesa de trabajo de Humboldt y Bonpland (Ette, 1994), los resultados que ambos obtuvieron de su expedición en América fueron a su vez puestos en escena durante los movimientos independentistas americanos, utilizados por muchos de los gobiernos en procesos emancipadores. Cada país realizó su propia interpretación y edificó su propia imagen de Humboldt. Cada país americano que visitó el famoso erudito parece crear a su propio Humboldt y por lo tanto nacionalizar su herencia (Rebok, 2019).

Por otra parte, los dos viajeros fueron sensibilizados a las ideas liberales que circulaban en América. Hubo probablemente una "criollización" de sus ideas y de su discurso, no solamente intercambiando con las élites, sino con todo el pueblo, enriqueciendo su pensamiento. Se puede leer perfectamente en el análisis que Humboldt realizó sobre Nueva España, lo cual coincidía con el desarrollo económico autónomo que pedían los criollos (Humboldt, 1811).

También se podría poner de relieve una "bonplandeanización" pues Aimé Bonpland se acercó e intercambió con muchos americanos, aunque a diferencia de Humboldt no dejó huellas documentadas de su expedición. Pero Bonpland fue formado en el Museo Nacional de Historia Natural de París dentro de un clima revolucionario extremo. En efecto, Bonpland había experimentado la Revolución Francesa primero en La Rochelle, donde vivía en el año 1789. Después estudió medicina y botánica a partir de 1793 en París, durante el gobierno de "terror" dirigido por Robespierre. De hecho, Bonpland fue mucho más sensible a las reivindicaciones de los americanos y más radical que Humboldt.

Haría falta llevar a cabo otras investigaciones para determinar en lo posible los intercambios, las interacciones intelectuales que existieron entre dos hombres que vivieron juntos durante seis años. No fue estudiada hasta ahora la genealogía de su pensamiento al respecto como tampoco la influencia que pudo haber tenido Bonpland en el pensamiento de Humboldt. $\mathrm{Si}$ algunos americanos pudieron influir sobre el pensamiento del Prusiano, la mudez de Bonpland durante el viaje es impresionante: ningún diario, pocas cartas, casi nada escrito de puño y letra del francés que permita apreciar la influencia del francés.

Mientras Humboldt desarrollaba su geografía política, Bonpland llevó a cabo una tarea científica enorme por lo que se refiere a la parte botánica. El homenaje de Humboldt al respecto es conocido, pues en el año 1805 
escribió que durante "su" expedición, fue Bonpland quien se encargó de describir la gran mayoría de las plantas, exactamente el 99\% de las descripciones botánicas, las cuales eran perfectas. Además, Bonpland numeró cada muestra que recolectaron, idea totalmente nueva para la época (Stauffer, 2019).

Pero describir no es clasificar y al principio del siglo XIX varios sistemas de clasificación coexistían. Para clasificar se necesitaban entonces otras exploraciones, se debía apoyar con la revisión en los herbarios existentes y con los libros de botánica presentes en el "Muséum National d'Histoire Naturelle", a fin de identificar precisamente cada muestra. Ahora bien, las muestras llegaban a un total de 60.000. Es decir, que después del trabajo de terreno descriptivo, faltaban aún años de trabajo para clasificar la totalidad de las muestras colectadas entre 1799 y 1804 .

Bonpland eligió un sistema de clasificación mixto basándose en la nomenclatura de Linné, con un poco de Jussieu y de Lamarck (Hamy, 1906). Pero no acabó el trabajo pues la cooperación entre Humboldt y Bonpland se extinguió poco a poco, adoleciendo del problema de la autoridad científica, Humboldt no habiendo sido al parecer tan agradecido con el trabajo de terreno que llevó a cabo su compañero.

Desde 1805, mientras Bonpland corregía los manuscritos de Humboldt y redactaba la parte botánica del viaje, otro botánico amigo de Humboldt, Carl Ludwig Willdenow, publicó la descripción de un nuevo espécimen de planta que Bonpland quería publicar él mismo. Humboldt juró a Bonpland no estar al tanto, de haber permitido a Willdenow describir cuatro o cinco especies de plantas enviadas desde Cuba en el año 1801. Sin embargo, en una carta escrita desde La Habana en 1801 a Willdenow, Humboldt autorizaba a su compatriota, en su nombre y en el nombre de Bonpland, en elegir entre los especímenes que deseaba, dejándole la primacía del descubrimiento y la autoridad científica. Instaba a Willdenow a que no use muchas plantas, no por razones éticas sino técnicas, ya que las muestras enviadas eran de menor calidad que las muestras que Bonpland y Humboldt llevaban con ellos (Hamy, 1905; Cerruti, 2012).
La noción de autoridad científica fue fundamental para Bonpland. Después de 1816, defendió dos veces la anterioridad de su descubrimiento en contra de dos botánicos. En 1832, Bonpland se enteró que Auguste de Saint-Hilaire había clasificado y publicado la yerba mate o Ilex paraguayensis. Bonpland le acusó de mentira y reivindicó el descubrimiento y la clasificación, a tal punto que hoy en día Bonpland comparte con Saint-Hilaire la autoridad de la atribución de esa planta. Unos años más tarde, en 1838, Bonpland escribió una memoria cuando se dio cuenta que el "maíz del agua" que había estudiado desde el año 1820 había sido publicado bajo el nombre de Victoria regia por Charles Waterton.

También se hubiese podido atribuir a Bonpland la autoridad científica sobre varios descubrimientos en el campo taxonómico pues, como lo escribió Humboldt a Bonpland en 1811 , se lo merecía respecto al trabajo de anatomía que el francés había hecho en el terreno (Cerruti, 2012). Pero esa vez tampoco se concretó.

Bonpland no obtuvo tampoco el reconocimiento que esperaba cuando intentó desarrollar su propia expedición científica, buscando el apoyo del "Muséum National d'Histoire Naturelle" y del gobierno francés, sin éxito. Hasta 1808, el francés intentó ingresar a esa estructura científica como es la Academia de las Ciencias de París, pero tampoco tuvo éxito. Así, el viaje no le otorgó inmediata y automáticamente una mejor situación ni menos una fama en el centro científico europeo. Una frase de Humboldt a propósito de Bonpland escrita más tarde resumió muy bien la situación de Bonpland: solo es un sabio quien viajó para colectar buenas hierbas (Hamy, 1906).

Así fue, si bien Aimé Bonpland ingresó a Malmaison y Navarre como botánico y responsable de las propiedades de la emperatriz Josefina desde 1808 hasta 1814 , se quedó a la sombra o al margen de las grandes instituciones científicas francesas. En efecto, dirigió un laboratorio vegetal y animal de alto nivel, pero perteneciente a un particular y por eso al margen, y casi en competencia, con las instituciones científicas gubernamentales. Después de la muerte de Josefina en 1814 el laboratorio periclitó. 
En 1816, cuando Bonpland volvió a viajar a América esta vez definitivamente, Humboldt confió la clasificación de los herbarios al botánico berlinés Carl Sigismund Kunth quien pasó el resto de su vida clasificando, mientras Bonpland volvió a hacer lo que más le gustaba, es decir explorar y describir. Quedaría por resolver el problema de la autoridad de atribución de cada planta entre Bonpland y Kunth, porque hasta ahora todas las clasificaciones están atribuidas a Kunth. Sin embargo, la autoridad científica de cada descubrimiento botánico es fundamental para discernir, medir y valorar la participación de Bonpland en su dimensión científica. Por eso, habría que explorar el herbario general del "Muséum National d'Histoire Naturelle", y estudiar caso por caso (Stauffer, 2019).

\section{Infelices coincidencias (1816-1858)}

La decisión de Bonpland de venir más precisamente al ex virreinato del Río de la Plata nuevamente independiente fue guiada a través de la propuesta hecha por las autoridades independentistas de Buenos Aires de transferir los saberes, los métodos y los laboratorios europeos para fomentar su propia tradición científica. El proyecto era de crear un jardín botánico y un gabinete de historia natural en Buenos Aires según los modelos de "Malmaison" y del "Muséum National d'Histoire Naturelle". Se necesitaba además de un trabajo previo de exploración y de recolección. Era una ocasión única para no integrar sino crear una institución científica.

También era una oportunidad para llegar un poco más a la altura de Humboldt, no como teórico, pues Humboldt se sitúa a unas alturas innegables, sino como descriptor y como otro nuevo descubridor de América. Completar el viaje efectuado con Humboldt podía convertir a Bonpland en un "tercer descubridor de América" en vez de quedarse como secretario de su compañero de viaje, pues desde 1799 hasta el final de la expedición Aimé Bonpland fue considerado y presentado en parte por la prensa ni siquiera como el botánico del viaje, sino como el secretario de Humboldt (PuigSamper et Rebok, 2002; Cala, 2011).

La elección de Bonpland en irse a Buenos Aires contiene una dimensión política importante pues marcó su voluntad de participar de manera activa en el movimiento independentista como su diferencia y su independencia respecto de Humboldt. En efecto, para Humboldt la expedición llevada a cabo no representa más que una etapa en sus investigaciones. Sin duda, las obras de Humboldt han influido ampliamente en la concepción del mundo americano y han contribuido a modificar la imagen que se tenía del Nuevo Mundo en la Europa del siglo XIX (Minguet, 1969, 1974; Rebok, 2003).

Humboldt formaría parte de la "disputa del Nuevo Mundo" al criticar y refutar los reiterados prejuicios eurocéntricos tanto en el campo natural como moral. Pero el prusiano no creía en la posibilidad de un movimiento de independencia, y temía que los partidarios de la separación no serían capaces de realizar una sociedad estable como las de Europa o Norteamérica. Como reformista, pensaba que se podía salvar el imperio integrando los criollos dentro del ámbito español imperial (Gerbi, 1955; Minguet, 1974).

Aimé Bonpland, por su parte, estuvo a favor de las independencias y desarrolló vínculos para favorecer a los independentistas. Desde su regreso a Francia en 1804, Bonpland vio en Simón Bolívar un posible líder, visto que los acontecimientos hacen que surjan los dirigentes mientras Humboldt era muy escéptico en las capacidades políticas del futuro Libertador. Entre 1808 y 1816, desde la invasión de España por Napoleón I hasta las primeras proclamaciones independentistas, Bonpland propuso dos veces ir hacia América para apoyar a los insurgentes y fomentar vínculos políticos entre ellos y su país (Cerruti, 2012).

Prefiriendo los actos en vez de los discursos, Bonpland se puso en contacto directo con los independentistas para hacer "ciencia política", apoyando a partir de 1814 los proyectos de los libertadores quienes afirmaban que había que librarse del oscurantismo colonial (Leguizamón, 1940). Convencido por las autoridades de Buenos Aires donde llegó a principios de 1817, Bonpland empezó su exploración en 1820 hacia las antiguas misiones jesuíticas del Paraguay pues les parecía las más fértiles científicamente como económicamente. 
Pero el botánico se encontró en medio de una región conformada por las nuevas entidades políticas. Considerado como un agente de las autoridades correntinas mientras desarrollaba una estructura agrícola en la frontera inestable paraguaya-correntina, fue raptado y exiliado por las fuerzas paraguayas en la misión de Santa María de Fe desde 1821 hasta 1831.

Durante ese periodo, el francés no pudo hacer ciencia ni menos política, sino agricultura y medicina. La decisión del dictador del Paraguay José Gaspar Rodríguez de Francia en ubicarle en una misión se puede interpretar como la voluntad de obtener beneficios de sus conocimientos en esas áreas. Los proyectos del doctor Francia coincidían con los del francés, ambos desarrollando el policultivo, el establecimiento de Bonpland convirtiéndose en una "granja modelo". Además, hizo aprender y aprendió medicina al contacto de la población guaraní (Boccia Romañach, 1999). Por fin, parece que su patriotismo y bonapartismo creció y se idealizó.

Los experimentos de Bonpland en Paraguay fueron la base de sus empresas futuras pues volvió a intentar en Brasil y en Argentina lo que consiguió hacer en Paraguay. Durante la década del 1830 abandonó sus objetivos científicos iniciales, rechazando varias oportunidades para participar en las estructuras científicas suramericanas. Al contrario, se consagró cada vez más a la medicina, a la agricultura y a la política, buscando a un hombre providencial a imagen y semejanza de Napoleón I capaz de unificar las provincias argentinas y permitir un acercamiento con Francia.

Esa sensibilidad americanista se fundió en cuatro representaciones. En primer lugar la idea que la base de la civilización de esa parte de América era basada en el interior del país, lo cual era una fuente inagotable de riquezas. En segundo lugar, la convicción que hacía falta integrar los pueblos autóctonos en el proceso de desarrollo del país. Luego, la idea que Francia tenía que participar en ese desarrollo pues su país, según Bonpland, tenía una misión "civilizadora". En efecto, hubo un ámbito en lo cual Bonpland nunca dudó, fue su patriotismo, característica fundamental de una gran mayoría de los viajeros quienes, tal Ulloa o Ulises, llevaron con ellos su amor al país. Por fin, la necesidad de encontrar en América a una persona capaz de ser un vínculo, es decir un hombre providencial que pueda trasmitir dicha "civilización" entre el "centro" europeo y la "periferia" americana (Cerruti, 2012).

Esa sensibilidad se solidificó durante las décadas 1840 y 1850 pues los ríos de la Mesopotamia argentina eran percibidos como una arteria y un vínculo entre las provincias, como con Brasil, Uruguay y, finalmente, Europa. También, en un contexto de guerras civiles, Bonpland cambió su postura, de científico y observador al de actor pues, además actuó como médico al servicio de los ejércitos que combatían cuando Juan Manuel de Rosas llevó a cabo varias misiones diplomáticas a favor del gobierno de Corrientes. Los gobernadores correntinos Pedro Ferré y Juan Pujol encarnaron, para el sabio, el prototipo del buen gobierno por su francofilia, su apertura a las ideas europeas y sus leyes favoreciendo a los extranjeros.

Queda por analizar el porqué de sus tentativas de viajes, de transferencia y de intercambios científicos no fueron exitosas. En primer lugar, pesó el contexto político. En efecto hizo falta esperar la segunda parte del siglo XIX para que la región se estabilice y que los gobernantes puedan llevar a cabo una política científica perdurable. En segundo lugar, no pesó sino faltó, dentro del contexto científico, un impulso y un respaldo institucional. A pesar que existían intelectuales y científicos activos en el Río de la Plata el problema fue que, en materia de ciencias naturales, los gobernantes no estructuraron ni tampoco apoyaron de manera significativa los esfuerzos individuales, como para consolidar una ciencia nacional. La cultura científica rioplatense conoció después de la independencia un proceso de militarización y una carencia de institucionalización, a diferencia de lo que ocurría en países como México (Montserrat, 2000; de Asúa, 2010).

Otro elemento de análisis deriva del precedente. En efecto, durante la década 1830, Bonpland intentó salir de su aislamiento científico intentando posicionarse de nuevo hacia el centro científico europeo. Intentó volver a trabajar directamente con Francia para promulgar su autoridad científica. Sin 
embargo, se enfrentó a varios obstáculos. El primero fue la distancia y las dificultades de las comunicaciones trasatlánticas, pues podían pasar años entre el envío de material científico y la respuesta de sus corresponsales en Europa. Aquí existe un conflicto evidente entre el investigador y el centro científico. Además, Bonpland nunca estuvo en posición de exigir o de obtener que se publiquen sus envíos o que se realicen estudios sobre las muestras que mandó a Europa. Como muchos otros científicos periféricos, sufrió el hecho de que muchos viajeros europeos usaron material o informaciones sin intentar consolidar las redes científicas trasatlánticas ni proponer una cooperación paritaria.

Un obstáculo vinculado con los anteriores fue la falta de interés por los aportes científicos de Bonpland, quien fue considerado, como lo había presumido Humboldt, como "un sabio quien viajó para colectar buenas hierbas". Fue considerado como un corresponsal periférico, muy lejos de lo que el viajero francés esperaba. A ese propósito, se puede comparar la trayectoria de Bonpland con la de Alcides d'Orbigny quien tampoco obtuvo el reconocimiento esperado, aunque publicó en Francia durante trece años sus investigaciones y sus aportes científicos fueron considerados como primordiales. El latino-americanismo como ideología apareció durante la segunda mitad del siglo XIX en Francia y el americanismo como ciencia fue oficializado en el año 1875 durante la celebración de su primer congreso, cuando empezaron y se desarrollaron las ciencias antropológicas y paleontológicas (Chonchol et Martinière, 1985; Riviale, 1996; Logie et Riviale, 2009).

Esos obstáculos desaparecieron cuando, a partir de las primeras exposiciones universales, a principios de la segunda mitad del siglo XIX, la oferta y la demanda científicas trasatlánticas evolucionaron y coincidieron (López Ocón et al., 2005). Los estándares elaborados en los laboratorios científicos del centro europeo fueron reapropiados y utilizados para sostener un proyecto político que tenía como meta valorar el patrimonio cultural, natural y sobre todo económico de los países del Cono Sur. En ese periodo y más especialmente en Argentina, después del gobierno de Juan Manuel de
Rosas, el país se abrió nueva e ideológicamente a Europa. Los museos de historia natural cobraron entonces una función de propaganda, poniendo de relieve las riquezas naturales del país hacia Europa para favorecer la inmigración (Cerruti, 2012).

Falta presentar el último elemento de análisis, el elemento humano es decir Bonpland mismo. Los "atermoiements" de Bonpland, es decir sus dilaciones, le llevaron a ponerse a él mismo en una posición periférica. En efecto, Bonpland dudó en concretar sus viajes científicos; dudó en elegir entre una vida científica y otra de terrateniente, estando en una posición ineficaz científicamente; dudó en aceptar varias oportunidades científicas para ingresar o fomentar centros científicos en América. Esa dimensión humana y psicológica es por cierto importante también para entender mejor la relación científica, política y cultural entre Aimé Bonpland y las Américas.

\section{Conclusiones}

Aimé Bonpland formó parte de los muchos europeos quienes, durante la primera mitad del siglo XIX, intentaron participar del movimiento de desarrollo de las Américas. El francés quiso aprovechar su primera experiencia americana, basada en su experiencia en Malmaison y Navarre, para construir y fomentar en el Río de la Plata un laboratorio y una tradición científica. Primeramente, con un enfoque universal siguiendo la tradición ilustrada del siglo XVIII, pero con un enfoque más regional y geocéntrico después de que el viajero se trasformó en un inmigrante, enfocándose en prioridad en la puesta en valor y en escena de los recursos rioplatenses.

La inestabilidad política como la marginalización de las ciencias naturales por ambos lados del océano Atlántico impidió el desarrollo de intercambios eficaces. La curiosidad que pudo despertar las investigaciones de Bonpland en Europa fue insuficiente para fomentar un saber interdisciplinar como una comunidad científica internacional característica del americanismo. Al final de la Guerra Grande en 1852, Bonpland consagró los últimos años de su vida en perdurar 
sus trabajos agrícolas como científicos a través del apoyo de las autoridades de la provincia de Corrientes. Encontró entonces un equilibrio entre ambas actividades.

Consciente de su aislamiento y de su retraso científico respecto a Europa, consciente y orgulloso de su integración y de su enraizamiento en esa parte de las Américas, frontera de varios países, y realizando varias actividades, Bonpland da testimonio de los esfuerzos que se hicieron para poner de relieve los recursos naturales, como del compromiso de un investigador, de un patriota y de un independentista en materia económica como política y social. Ahora su experiencia que fue sometida a felices o infelices coincidencias pide comparaciones con otras, para obtener un panorama más general de las acciones e interacciones transatlánticas en esa época.

\section{Agradecimientos}

A Myriam Carolina Peichoto, María Cecilia Puigbó y María Silvia Ferrucci por su apoyo. A Aurora Arbelo, Cécile Aupic, Stephen Bell, David Guevara, Irene Prüfer Leske, Gladis Mango, Diana Reyes, Melina Riabis, Lucía Rossi y Fred Stauffer por haber compartido sus saberes y enriquecido mis conocimientos.

\section{Bibliografía}

BOCCIA ROMANACH, A. (1999). Amado Bonpland Carai Arandu. El Lector 1, Asunción, Paraguay.

CALA, P. (2011). Una visión histórica de la ictiología, con especial énfasis en América y Colombia. Dahlia 11: 75-125.

CERRUTI, C. (2012). L'américanisme en construction. Une pré-histoire de la discipline d'après l'expérience du naturaliste Aimé Bonpland (1773-1858). Doctorado en Historia. Universidad de La Rochelle. $998 \mathrm{pp}$.

CHONCHOL, J. \& MARTINIÈRE, G. (1985). L'Amérique latine et le latino-américanisme en France. L'Harmattan, Paris. https://doi.org/10.4000/books.iheal.3345

DE ASÚA, M. (2010). La ciencia de Mayo: la cultura científica en el Río de la Plata, 1800-1820. Fondo de Cultura Económica, Buenos Aires.
ETTE, O. (1994). La puesta en escena de la mesa de trabajo en Raynal y Humboldt. Cuadernos americanos 46: 29-68.

GERBI, A. (1955). La disputa del nuovo mondo. Storia di una polémica, 1750-1900. Riccardo Ricciardi Editore, Milan.

GUERRERO, C. (dir.) (2019). Humboldt y Bonpland en Ecuador: un viaje que cambió el mundo. Ñan 34: 1-129.

HAMPE MARTÍNEZ, T. (2002). Carlos Montúfar y Larrea (1780-1816), el quiteño compañero de Humboldt. Revista de Indias LXII: 711-720.

HAMY, T. J. E. (1905). Lettres américaines d'Alexandre de Humboldt (1798-1807). Précédées d'une notice de J.C. Delamétherie et suivies d'un choix de documents en partie inédits. E. Guilmoto, París.

HAMY, T. J. E. (1906). Aimé Bonpland, médecin et naturaliste, explorateur de l'Amérique du Sud; sa vie, son oeuvre, sa correspondance avec un choix de pièces relatives à sa biographie, un portrait et une carte. E. Guilmoto, París. https://doi.org/10.5962/bhl.title.60911

HUMBOLDT, A. (1811). Essai politique sur le royaume de la Nouvelle Espagne. F. Schoell, París. https://doi.org/10.5962/bhl.title.139051

LEGUIZAMÓN, G. (1940). Archivo de Bonpland, tomo IV: Londres, cuartel general europeo de los patriotas de la emancipación americana. Trabajos del Instituto de Botánica y Farmacología "Julio A. Roca" II (2). Imprenta y Casa Editora Coni, Buenos Aires. 52 pp.

LOGIE, E. \& RIVIALE, P. (2009). Le congrès des américanistes de Nancy en 1875: entre succès et désillusions. Journal de la société des américanistes 95: 151-171.

https://doi.org/10.4000/jsa.11036

LÓPEZ OCÓN, L., CHAUMEIL, J. P. \& VERDE CASANOVA, A. (eds.). (2005). Los americanistas del siglo XIX. La construcción de una comunidad internacional. Iberoamericana \& Vervuert, Madrid \& Frankfurt. https://doi.org/10.31819/9783964565471

MINGUET, C. (1969). Alexandre de Humboldt, historien et géographe de l'Amérique espagnole. Maspéro, París. https://doi.org/10.4000/books.iheal.3660

MINGUET, C. (1974). La América de Humboldt. Boletín AEPE 10: 53-60.

MONTSERRAT, M. (comp.). (2000). La ciencia en la Argentina entre siglos. Textos, contextos e instituciones. Manantial, Buenos Aires.

PUIG-SAMPER, M. Á \& REBOK, S. (2002). Un sabio en la meseta: el viaje de Alejandro de Humboldt 
a España en 1799. Revista de Occidente 254-255: 95-106.

REBOK, S. (2003). La expedición americana de Alexander von Humboldt y su contribución a la ciencia del siglo XIX. Bulletin de l'Institut français d'études andines 32: 441-458. https://doi.org/10.4000/bifea.6080

REBOK, S. (2019). Percepción de Humboldt en Iberoamérica: Retos y oportunidades de una temporada temática. Institut für Auslandsbeziehungen, Stuttgart.
RIVIALE, P. (1996). Un siècle d'archéologie au Pérou (1821-1914). L'Harmattan, París.

STAUFFER, F. (2019). Contribución de Humboldt y Bonpland al conocimiento botánico Neotropical: un estudio basado en especímenes, archivos y manuscritos originales. III Congreso internacional e interdisciplinar Alexander von Humboldt - Aimé Bonpland. Asunción.

ZEUSKE, M. (2003). ¿Humboldteanización del mundo occidental? La importancia del viaje de Humboldt para Europa y América Latina. Revista internacional de estudios humboldtianos IV: 3-28. 
